\title{
A two-stage stochastic programming approach for reliability constrained power system expansion planning
}

\author{
Meltem Peker, Ayse Selin Kocaman*, Bahar Yetis Kara \\ Industrial Engineering, Bilkent University, 06800, Ankara, Turkey
}

\section{A R T I C L E I N F O}

\section{Keywords:}

Filtering

Generation and transmission expansion

planning

Reliability

Stochastic programming

Transmission switching

\begin{abstract}
A B S T R A C T
Probabilistic realizations of outages and their effects on the operational costs are highly overlooked aspects in power system expansion planning. Since the effect of randomness in contingencies can be more prominent especially when transmission switching is considered, in this paper we introduce contingency-dependent transmission switching concept to ensure N-1 criterion. To include randomness of outages and the outputs (i.e. flow on the lines/generation amounts) during the outages, we represent each contingency by a single scenario. Status of transmission lines, generation amounts and power flow decisions are defined as recourse actions of our two-stage stochastic model, therefore, expected operational cost during the contingencies are taken into account in a more accurate manner. A solution methodology with a filtering technique is also proposed to overcome the computational burden. The model and the solution methodology are tested on the IEEE Reliability Test System and IEEE 118-bus power system and the results show that the solution method finds the solutions for these power systems in significantly shorter solution times. The solution method is also tested on a new data set for the 380$\mathrm{kV}$ Turkish transmission network. Suggestions for possible extensions of the problem and the modifications of the solution approach to handle these extensions are also discussed.
\end{abstract}

\section{Introduction}

Power system expansion planning determines the least costly expansion plan by locating new generation units and transmission lines. Generation expansion planning (GEP), a problem in which the location, capacity and time of building new power plants is determined [1-4], and transmission expansion planning (TEP), a problem that optimizes the design of a network by selecting the location, capacity and time of building new transmission lines [5-7] have been studied in this field. Generation-transmission expansion planning (GTEP) problem uses the interconnected nature of GEP and TEP problems (i.e. capacity and location of a new generation plant is affected by the available capacity of transmission lines) to simultaneously optimize them. For more information on the different modelling approaches and solutions techniques refer to $[8,9]$.

Reliability constrained GTEP (R-GTEP), another problem commonly studied in literature, defines reliability as the ability to withstand disturbances arising from outage of generation units or transmission lines [10]. The problem determines the new investments to guarantee that the system remains feasible in case of a component break-down (whole system load can still be met). Most of the studies that consider reliability criteria plan new investments based on only the feasibility of the power system after a line or generator contingency and ignore the outcomes during the contingency states [11-16]. As the probabilistic realizations of outages are customarily overlooked, the effect of randomness in contingencies on the investment plans and the cost of the expansion plans are usually disregarded. Some studies partially consider the probabilistic realization of outages by considering loss of load probability (LOLP) and/or expected energy not served (EENS) in the system [17-22] or risk-based decision-making process [23,24]. Although these studies consider the effect of randomness in contingencies on the investment costs, they still overlook the effect of probabilistic nature of contingencies on the operational costs. Reliability of power system has been also discussed considering different sources of uncertainties such as uncertainty in generation or consumer behavior of electricity price [25-27]. However, these studies also do not explicitly include the operational costs during the contingency states.

The role of randomness in outages in the power system expansion planning can be more prominent especially when transmission switching (TS) is considered. TS identifies the branches that should be taken out of service to change the topology of the system in order to increase the utilization of the network, decrease the total cost in the system and prevent the overloads on the transmission lines [28]. Beneficial impact of TS on the reliability and market efficiency of power system has been

\footnotetext{
* Corresponding author.

E-mail address: selin.kocaman@bilkent.edu.tr (A.S. Kocaman).
} 


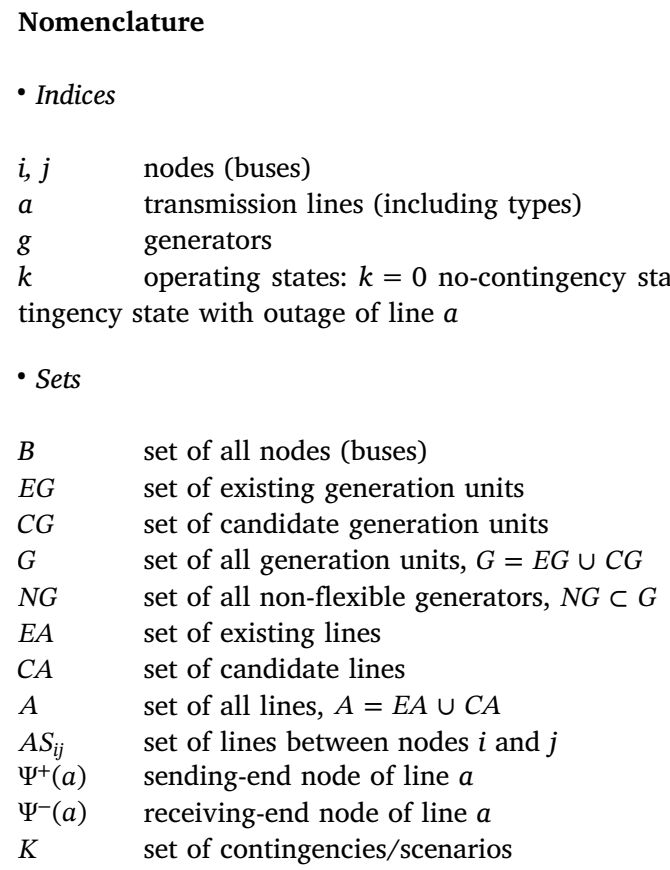

\section{- Parameters}

$\begin{array}{ll}l_{i} & \begin{array}{l}\text { demand of node } i(\mathrm{MW}) \\ \bar{F}_{a}\end{array} \\ \bar{G}_{i g} & \text { capacity of line } a(\mathrm{MW}) \\ G_{i g} & \text { minimum generation from unit } g \text { in node } i(\mathrm{MW}) \\ c_{g}^{i n v} & \text { annualized inver cost of unit } g(\$) \\ c_{g}^{\text {om }} & \text { operation cost of unit } g(\$ / \mathrm{MW} \mathrm{h}) \\ c f_{g} & \text { capacity factor of unit } g \\ c_{a}^{\text {line }} & \text { annualized inv. cost of line } a(\$) \\ \varphi_{a} & \text { susceptance of line } a(\mathrm{p} . \mathrm{u} .) \\ \sigma_{a} & \text { forced outage rate of line } a \\ \Gamma_{a}^{k} & 1, \text { if line } a \text { is on under contingency } k, 0, \text { if it is off } \\ p^{k} & \text { probability of contingency } k \\ d u r & \text { duration of the planning horizon }\end{array}$

- Decision variables

$\begin{array}{ll}X_{i g} & 1 \text { if unit } g \text { is built at node } i, 0 \text { o.w. } \\ P_{i g}^{k} & \text { generation of unit } g \text { in node } i \text { under contingency } k . \\ L_{a} & 1 \text { if line } a \text { is built, } 0 \text { o.w. } \\ S_{a}^{k} & 1 \text { if line } a \text { is closed under contingency } k \text { and } 0, \text { if it is open } \\ f_{a}^{k} & \text { power flow on line } a \text { under contingency } k \\ \theta_{i}^{k} & \text { voltage angle of node } i \text { under contingency } k\end{array}$

demonstrated in academic studies as well as industrial applications. In $[29,30]$, the authors discuss the congestions and cost savings of TS for the PJM system for different voltage levels changing from $115 \mathrm{kV}$ to $765 \mathrm{kV}$. Furthermore, in [31], examples from California ISO, Independent System Operator of New England and PJM demonstrate that some protocols for switching transmission lines are applied for decreasing congestion, maintenance scheduling or seasonal switching.

The value of TS is also discussed for theoretical examples as in [32-35]. In [32], the authors use switching operations in expansion planning problem and discuss the effect of TS on expansion planning decisions. In [33], the value of a seasonal transmission switching on the total cost and reliability level of the power system is discussed and in [34], short-circuit current limitations for a power system considering TS and N-1 reliability criterion is analyzed. In [35], the authors analyze the effect of TS on the power system and they calculate the monetary value of EENS for the solutions and discuss the effect of TS on EENS. However, most of the studies determine the status of transmission lines before observing the contingencies and network topology is designed to satisfy the whole system load after any contingency without requiring operator control on generators. This approach is referred to as preventive security constrained transmission switching [35], and ignores the probabilistic nature of outages and the expected operational costs during the contingencies. Therefore, the overall costs of the investment planning projects are underestimated. Although having a single network topology for all time periods is extremely unlikely due to uncertainties [31], system operators have flexibility to monitor and change the status of the transmission lines after a contingency.

Considering the operational costs during the contingency states and changing the network topology for each contingency can affect the reliability of the power system and the investment plans significantly. Especially for power systems that have flexible generators, after a line or generator outage, corrective actions such as changing the outputs of the flexible generators and network topology by switching transmission lines can be taken to address the contingency [36]. Thus, over investments (i.e. building new generation units to supply only peak loads) can be prevented and utilization of the system can be increased. For this purpose, we introduce a new transmission switching concept, contingency-dependent TS, which entails the definition of transmission switching decisions based on each contingency. This concept is made possible by means of our two-stage stochastic programming model where the power flows, status of transmission lines and the generation amounts are defined as recourse actions. As generation amounts for each contingency state can be different from each other, the expected value of the operational costs of during the contingency states are taken into account in a more accurate manner.

Literature utilizes different approaches to overcome computational burden of the R-GTEP problem such as determining a short list of the candidate lines [12,13], line outage distribution factors (LODFs) method [14,15], worst case analysis [16] and umbrella constraint discovery (UCD) technique $[37,38]$. This paper presents a computationally tractable solution approach that includes a filtering to find the contingencies that do not affect the power system's reliability. This solution approach decreases the number of contingencies considerably and temporarily eliminates the constraints related with that contingencies. A similar notion is also used in UCD technique discussed in $[37,38]$ to identify redundant constraints in security-constrained optimal power flow (SCOPF) and security-constrained unit commitment (SCUC) problems, respectively. Although the benefits of UCD technique is verified on both problems, it is discussed that the proportion of non-umbrella constraints in SCUC is lower compared to the proportion of non-umbrella constraints in SCOPF. Since our problem is structurally closer to the SCUC problem, and includes more binary variables in nature (i.e. decisions for the investment planning and switching transmission lines for each contingency) than the binary variables in SCUC problem, in this study, we utilize a filtering approach to find the critical contingencies. Thus, by using the filtering approach, we reduce the computational challenge of the two-stage stochastic programming model and find the optimal or near-optimal solutions for the original problem that satisfies the $\mathrm{N}-1$ reliability criterion.

This paper proposes a two-stage stochastic programming model for the R-GTEP problem that minimize investment and expected operational costs. Our two-stage stochastic model represent each contingency state by a single scenario with a probability of happening and includes operational costs during the scenarios in the expected form. The main contributions of this paper are listed below:

- We propose a two-stage stochastic programming model for the RGTEP problem that includes expected operational cost during the 
contingencies, an aspect that has been overlooked in the literature which can affect the investment plans.

- We calculate expected operational costs in a more accurate manner by utilizing the proposed contingency-dependent TS concept.

- We present a computationally tractable solution approach that includes a filtering technique.

- We discuss the possible extensions of the problem and modifications of the solution approach to handle these extensions.

In this study, the proposed model is applied to the IEEE 24-bus power system and the value of incorporating randomness of contingencies is discussed. We outline the effect of considering expected operational cost and the value of contingency-dependent TS by showing that the network topologies for each contingency are different from each other. We also discuss the computational performance of the proposed solution approach on a larger dataset by comparing the results of the model and the solution approach for the IEEE 118-bus power system. We then introduce a real-world data set to the literature for the $380-\mathrm{kV}$ Turkish transmission network and present our results for this data set.

In the next section, we present the mathematical model and explain the solution methodology for the problem. We then discuss the results on the IEEE 24-bus and IEEE 118-bus power systems for different instances in Section 3. We also present the dataset of the current Turkish transmission network and the solutions for this dataset in the same section. In Section 4, we discuss possible extensions of the problem and modifications to the proposed model and the solution approach to handle these extensions. This paper concludes with final remarks in Section 5 .

\section{CD-R-GTEP problem}

Contingency-dependent R-GTEP (CD-R-GTEP) problem determines the optimal expansion plan and optimal network configuration for each contingency that satisfies the required reliability level. We represent outage of each line as a single scenario with a certain probability of happening. As the operational cost in each scenario can be different due to unavailability of the line in that scenario, we propose a two-stage stochastic model to handle the probabilistic realization of outages. The first stage decisions of the proposed model include the investments of generation units and transmission lines. Power flows, generation amounts and status of transmission lines are recourse actions of the second-stage. For calculating the probability of scenarios, we utilize forced outage rate (FOR) of transmission lines and operational costs of scenarios are included in the objective function in the expected form. A set of generation technologies and a set of transmission lines with different properties are considered. As the problem in this study is complex and nonlinear, we use a DC network representation as customarily done in majority of the studies in this field i.e. [11-22,28,32].

\subsection{Mathematical model of CD-R-GTEP}

This section first presents the standard form of a two-stage stochastic programming model and then provides the extensive form of the model. In the standard form of a two-stage stochastic model, the first stage decisions are generally represented by $x$, and the second stage decision variables are represented by $y(\omega)$ for a realization of $\omega$ in the probability space $(\Omega, P)$. The standard form of a two-stage stochastic programming model is represented as:

$$
\begin{aligned}
& \operatorname{minc}^{T} x+\mathbf{E}_{\omega}[Q(x, \omega)] \\
& \text { s. } t A x=b \\
& \quad x \geqslant 0
\end{aligned}
$$

where $\quad Q(x, \omega)=\min _{y(\omega) \geqslant 0}\{q(\omega) y(\omega): T(\omega) x+W y(\omega)=h(\omega)\}$ and $\mathbf{E}_{\omega}[Q(x, \omega)]$ is the expected value of the second stage. With a finite number of second stage realizations, $S$, we obtain the extensive form of the two-stage model:

$$
\begin{array}{ll}
\operatorname{minc}^{T} x+\sum_{s=1}^{S} p_{s} q_{s} y_{s} & \\
\text { s. } t A x=b & \\
T_{s} x+W y_{s}=h_{s} \quad \forall s=1 \ldots S \\
x \geqslant 0, y_{s} \geqslant 0
\end{array}
$$

where $p_{s}$ is the probability of the scenario $s$ and $\sum_{s=1}^{S} p_{s} q_{s} y_{s}$ is the expectation of the second stage.

The following model (CD-TS) is the extensive form of the two-stage stochastic programming model for the problem where the first stage decisions include investment of assets and the second stage decisions are scenario-based operational decisions such as power flows, generation amounts and status of transmission lines (open/close). Each decision variable except $X_{i g}$ and $L_{a}$, given in the nomenclature, has a dimension $k$ to represent scenario-based operational decisions. Scenario $k=0$ represents the no-contingency state and scenarios $k>0$ represent a scenario associated with a contingency state with outage of a single line. The objective function of CD-TS is presented as follows:

$$
\begin{aligned}
\text { min } z_{\text {gen }} & +z_{\text {line }}+\sum_{k \in K} p^{k} z_{\text {om }}^{k} \\
z_{\text {gen }} & =\sum_{i \in B} \sum_{g \in C G} c_{g}^{i n v} X_{i g} \\
z_{\text {line }} & =\sum_{a \in C A} c_{a}^{\text {line }} L_{a} \\
z_{\text {om }}^{k} & =\sum_{i \in B} \sum_{g \in G} c_{g}^{\text {om }} c f_{g} d u r P_{i g}^{k}
\end{aligned}
$$

The objective function (1) minimizes the annual total cost. The first two terms are the annualized investment costs of the new generation units and new transmission lines, respectively. $z_{o m}^{k}$ is the operational cost of scenario $k$ and by multiplying the operational cost of scenario $k$ with its probability of happening, $p^{k}$, the expected operational cost of all scenarios is included in the objective function. The objective function is subject to following constraints:

- Power balance constraint:

$$
\sum_{g \in G} P_{i g}^{k}+\sum_{a \in A S_{i j}: \Psi^{-}(e)=i} f_{a}^{k}-\sum_{a \in A S_{i j}: \Psi^{+}(e)=i} f_{a}^{k}=l_{i} \quad \forall i \in B, \quad k \in K
$$

Eq. (2) enforces power balance at node $i$, under contingency state $k$ which includes generation from the existing and new sources, incoming/outgoing flows and demand. By satisfying the demand at each bus, for all scenarios, the required N-1 reliability level is satisfied.

- Generation dispatch constraints:

$\underline{G}_{i g} \leqslant P_{i g}^{k} \leqslant \bar{G}_{i g} \quad \forall i \in B, \quad g \in E G, k \in K$

$\underline{G}_{i g} X_{i g} \leqslant P_{i g}^{k} \leqslant \bar{G}_{i g} X_{i g} \quad \forall i \in B, \quad g \in C G, \quad k \in K$

$P_{i g}^{k_{a}}=P_{i g}^{0} \quad \forall i \in B, g \in N G, \quad k_{a} \in K$

The power generation under contingency $k$ for existing and new plants are limited by (3) and (3'), respectively; and they (i.e. flexible generators) can adjust their outputs based on their capacity limits under each contingency. Eq. (4) guarantees that the output of power generated at the non-flexible generators does not change with any line contingency thus, for these types of generators, the generation under any contingency is equivalent to the generation under no-contingency scenario.

- Network constraints:

$-\bar{F}_{a} \Gamma_{a}^{k} S_{a}^{k} \leqslant f_{a}^{k} \leqslant \bar{F}_{a} \Gamma_{a}^{k} S_{a}^{k} \quad \forall a \in A, k \in K$

$f_{a}^{k}=\varphi_{a} \Gamma_{a}^{k} S_{a}^{k}\left(\theta_{i}^{k}-\theta_{j}^{k}\right) \quad \forall a \in A S_{i j}, \quad k \in K$ 
$S_{a}^{k} \leqslant L_{a} \quad \forall a \in A, \quad k \in K$

We introduce a binary parameter $\Gamma_{a}^{k}$ which takes value 1 if line $a$ is on (in operation), under contingency $k$ and takes 0 if it is off. We also introduce a binary decision variable to handle the switching of transmission lines and $S_{a}^{k}$ is equal to 0 if the line $a$ is opened under contingency $k$, and 1 if the line is closed under this contingency. Eq. (5) enforces the power flow limitations on each line that depends on the scenarios and statuses of lines (on/off). If the line $a$ is off in scenario $k, \Gamma_{a}^{k}=0$, or it is opened, $S_{a}^{k}=0$, then (5) reduces to $f_{a}^{k}=0$ which is consistent as there cannot be flow on that line. In the other case, i.e. line $a$ is on and closed in scenario $k$, then constraint (5) sets the lower and upper bounds for the flow on line $a$. Eq. (6) defines the power flow on line $a$ for scenario $k$ as a function of voltage angles differences of buses. Similar discussions for the constraint (5) can also be deducted for the constraint (6): if the line $a$ is off or opened, then $f_{a}^{k}=0$, otherwise it is equal to DC representation of Kirchoff's law. We note here that, constraint (6) is nonlinear and we linearize this constraint below using a Big-M type linearization technique. Eq. (7) satisfies that a line can be on if the line already exists or is built.

- Domain constraints:

$-\pi \leqslant \theta_{i}^{k} \leqslant \pi \quad \forall i \in B, k \in K$

$\theta_{\text {ref }}^{k}=0 \quad \forall k \in K$

$L_{a}=1 \quad \forall a \in E A$

$X_{i g} \in\{0,1\}, P_{i g}^{k} \geqslant 0, \theta_{i}^{k}$ urs $\forall i \in B, \quad g \in G, \quad k \in K$

$L_{a} \in\{0,1\}, S_{a}^{k} \in\{0,1\}, f_{a}^{k}$ urs $\forall a \in A, k \in K$

Eq. (8) limits the voltage angles at every bus under each scenario and Eq. (9) is the reference point for voltage angle profile of buses. Eq. (10) represents the existing transmission lines. Eqs. (11) and (12) are the domains of the decision variables.

We remark that, Eq. (6) is nonlinear due to multiplication of decision variables $S_{a}^{k}$ and $\theta_{i}^{k}$. We linearize the equation by using a similar technique used in $[5,39]$. Two nonnegative flow variables, $f_{a}^{k+}$ and $f_{a}^{k-}$, each one representing one direction for the same line $a$ for scenario $k$, express the unrestricted variable $f_{a}^{k}$ as the difference between two nonnegative decision variables as follows:

$f_{a}^{k}=f_{a}^{k+}-f_{a}^{k-} \quad \forall a \in A, \quad k \in K$

Similarly, two nonnegative variables, $\Delta \theta_{a}^{k+}$ and $\Delta \theta_{a}^{k-}$ express the difference of voltage angles of buses $i$ and $j$ for scenario $k$ as follows:

$\theta_{i}^{k}-\theta_{j}^{k}=\Delta \theta_{a}^{k+}-\Delta \theta_{a}^{k-} \quad \forall a \in A S_{i j}, \quad k \in K$

By using Eqs. (13) and (14), Eq. (6) is linearized and replaced with the following Eqs. (15)-(18):

$f_{a}^{k+} \leqslant \varphi_{a} \Gamma_{a}^{k} \Delta \theta_{a}^{k+} \quad \forall a \in A S_{i j}, \quad k \in K$

$f_{a}^{k-} \leqslant \varphi_{a} \Gamma_{a}^{k} \Delta \theta_{a}^{k-} \quad \forall a \in A S_{i j}, \quad k \in K$

$f_{a}^{k+} \geqslant \varphi_{a} \Gamma_{a}^{k} \Delta \theta_{a}^{k+}-M_{a}\left(1-S_{a}^{k}\right) \quad \forall a \in A S_{i j}, \quad k \in K$

$f_{a}^{k-} \geqslant \varphi_{a} \Gamma_{a}^{k} \Delta \theta_{a}^{k-}-M_{a}\left(1-S_{a}^{k}\right) \quad \forall a \in A S_{i j}, \quad k \in K$

Eqs. (15)-(18) correctly linearize Eq. (6) for a sufficiently large positive number $M_{a}$ in the Eqs. (17) and (18) so that the new constraints does not cut any feasible solution if the line $a$ is open, and $M_{a}=2 \pi \varphi_{a}$ can be used for the proposed model. When line $a$ is open in scenario $k$ (i.e. $S_{a}^{k}=0$ ), Eqs. (17) and (18) become redundant as $f_{a}^{k+}$ and $f_{a}^{k-}$ are already greater than or equal to 0 . For this case, Eqs. (15) and (16) are also redundant and do no cut any feasible solution since $f_{a}^{k}$ is already equal to zero from Eq. (5). When $S_{a}^{k}=1$, Eqs. (15) and (17) reduce to $f_{a}^{k+}=\varphi_{a} \Gamma_{a}^{k} \Delta \theta_{a}^{k+}$ and Eqs. (16) and (18) reduce to $f_{a}^{k-}=\varphi_{a} \Gamma_{a}^{k} \Delta \theta_{a}^{k-}$. By using the equalities in Eqs. (13) and (14), we obtain $f_{a}^{k}=\varphi_{a} \Gamma_{a}^{k} \Delta \theta_{a}^{k}$ which is the same equation obtained from the Eq. (6) when $S_{a}^{k}=1$. Thus, adding Eqs. (15)-(18) and removing Eq. (6) linearize the proposed model CD-TS.

In this study, we consider the scenarios only for no-contingency and single-line contingency. To include the expected operational cost of scenarios to the objective function, we first define the probabilities of no-contingency and single-line contingency scenarios, which are shown in Eqs. (19) and (20), respectively. To define the probabilities, we use the binomial distribution and FOR of transmission lines, $\sigma_{a}$, to describe the unavailability of transmission lines [24]. Note that, we assume independent outage of lines (and ruling out the possibilities of events where one line outage leads to the outage of other lines) and consider only no-contingency and single-line contingency scenarios as in [20] due to high probabilities of these cases. However, one can easily consider N-m (loss of $m$ lines simultaneously, $m>1$ ) and include the corresponding scenarios to the model.

$p^{0}=\prod_{a \in A}\left(1-\sigma_{a} L_{a}\right)$

$p^{k}=\sigma_{k} L_{k} \prod_{a \in A: a \neq k}\left(1-\sigma_{a} L_{a}\right)$

We note that the objective function is still nonlinear and can be linearized by applying a similar technique used in [20]. However, the proposed solution methodology in this study does not require a linear objective function. Here, we update the number of contingency states (scenarios) and recalculate their probabilities if a new line is built to calculate a more accurate operational costs of contingencies. Next section describes an efficient solution methodology to solve the computationally complex CD-R-GTEP problem.

\subsection{A scenario reduction based solution methodology}

The number of lines and contingencies has a significant impact on the solution time of the model especially for a large-size network. Considering all the contingencies simultaneously may increase the size of the problem dramatically and may also lead to memory problems. However, most of the contingencies do not affect power systems' reliability in real world examples [14]. These observations motivate us using a filtering technique as in [14] to find the redundant contingencies where the reliability of the power system is still maintained after removing these contingencies from the consideration. A similar filtering technique has been also utilized in [15] to reduce the number of scenarios related to the uncertainties of renewable generation units. Unlike our study, $[14,15]$ do not consider operational costs during the contingency states. They only consider the lines such that their outage will cause overloads on the other lines (important lines). However, in our solution approach, addition to the important lines discussed in $[14,15]$, we should consider the remaining lines for analyzing the effect of randomness in outages on the power system expansion plans. Our proposed scenario reduction based solution methodology (SRB) is explained via the following steps:

Step 1: Check whether the existing network topology (i.e. without allowing new investments) is feasible or not for the no-contingency state, which is equivalent to checking feasibility of the model CD-TS for only scenario $k=0$. For the feasibility check, add the following constraints to CD-TS and set contingency list $K=\{0\}$.

$X_{i g}=0 \quad \forall i \in B, g \in C G$

$L_{a}=0 \quad \forall a \in C A$

If it is not feasible, solve the original model CD-TS (after removing Eqs. (21) and (22)) for only scenario $k=0$. Get the optimal solution of the model and update the sets based on the new investments obtained from the optimal solution: add new transmission lines and generation units to the sets of existing transmission lines, $E A$, and existing generation units, $E G$, respectively, and remove these new lines and new 
generation units from the sets of candidate transmission lines, $C A$, and candidate generation units, $C G$, respectively.

Step 2: Calculate the probabilities of contingency states for all lines using the following equations. Here, we only consider the scenarios associated with the existing lines in $E A$. Thus, we set probabilities of the scenarios for the candidate lines to 0 . We remind that in order to define the probabilities, we use the binomial distribution and FOR of transmission lines, which is denoted by $\sigma_{a}$.

$p^{0}=\prod_{a \in E A}\left(1-\sigma_{a}\right)$

$p^{s}=\sigma_{s} \prod_{a \in E A: a \neq s}\left(1-\sigma_{a}\right) \quad \forall s \in E A$

$p^{s}=0 \quad \forall s \in C A$

Step 3: Decompose the problem into number of scenarios whose probability calculated in Eqs. (23)-(25)) is larger than 0 and create $|E A|+1$ subproblems, $P_{0}, P_{1}, \ldots, P_{k}, \ldots, P_{|E A|}$ where $P_{0}$ corresponds to the updated network topology for scenario $k=0$ (i.e. no contingency state) and $P_{k}$ is the topology when the $k$ th line in the set $E A$, is out of service. In this step, all candidate lines are also considered as out of service. Let $F R\left(P_{k}\right)$ be the feasible region of the $k$ th subproblem. Check the feasibility of each subproblem for the network obtained at the end of Step 1. If the $k$ th subproblem is infeasible, (i.e. there does not exist any power dispatch that satisfies the load without requiring any new investments), define the $k$ th line as critical line and the corresponding contingency as critical contingency. If the $k$ th subproblem is feasible (i.e. there exists at least one solution that satisfies the load without requiring any new investments), then define the $k$ th line as non-critical line and the corresponding contingency as non-critical contingency. Let $C C$ be the set for the critical contingencies (or critical lines) and NCC be the set for the noncritical contingencies (or non-critical lines). CC and NCC are defined as follows:

$C C=\left\{k: F R\left(P_{k}\right)=\varnothing\right\}$

$N C C=\left\{k: F R\left(P_{k}\right) \neq \varnothing\right\}$

Step 4: Generate a new scenario, referred to as super scenario (ss) and the corresponding subproblem for this scenario. The feasible region of the new subproblem is the same with the feasible region of $P_{0}$ and the probability of this scenario is equal to the sum of the probabilities of all the scenarios in the set NCC: $p^{s s}=\sum_{k \in N C C} p^{k}$. We note here that, the new generation plants and/or new transmission lines that are built can also be used for the scenarios in the set NCC. Thus, for the correct capacity planning of the new plants and/or lines, we incorporate all the scenarios in the set NCC and hence the non-critical lines are considered in the planning process. Define contingency set, $K$, as the union of the scenarios in CC and super scenario, i.e. $K=\{k: k \in C C$ and $s s\}$. Solve the model CD-TS optimally with only the scenarios in the updated set $K$, get the optimal solution and find the required new investments. Report the expansion plan (new generation units and transmission lines), and investment costs $\left(z_{\text {gen }}, z_{\text {line }}\right)$.

Step 5: The super scenario may underestimate or overestimate the true operational costs for the scenarios in the set NCC due to having different feasible region than $P_{0}$. Thus, in this step, the expected operational cost for all the scenarios is recalculated. Using the solution obtained at the end of Step 4, update the sets of the existing and new transmission lines, $E A, C A$, and sets of existing and new generation units $E G, C G$ using the same arguments as in Step 1. Update also the probabilities of the scenarios, $p^{0}$ and $p^{s} \forall s \in E A$ using the Eqs. (23)-(25) as probabilities may change with the new set of the existing lines, $E A$. Define contingency set, $K$ as the union of all scenarios in CC and NCC such that $K=\{k: k \in C C$ and $k \in N C C\}$. Solve the proposed model without allowing new expansions and add Eqs. (21) and (22) to the CD-TS. Solve the proposed model CD-TS with the new contingency set.

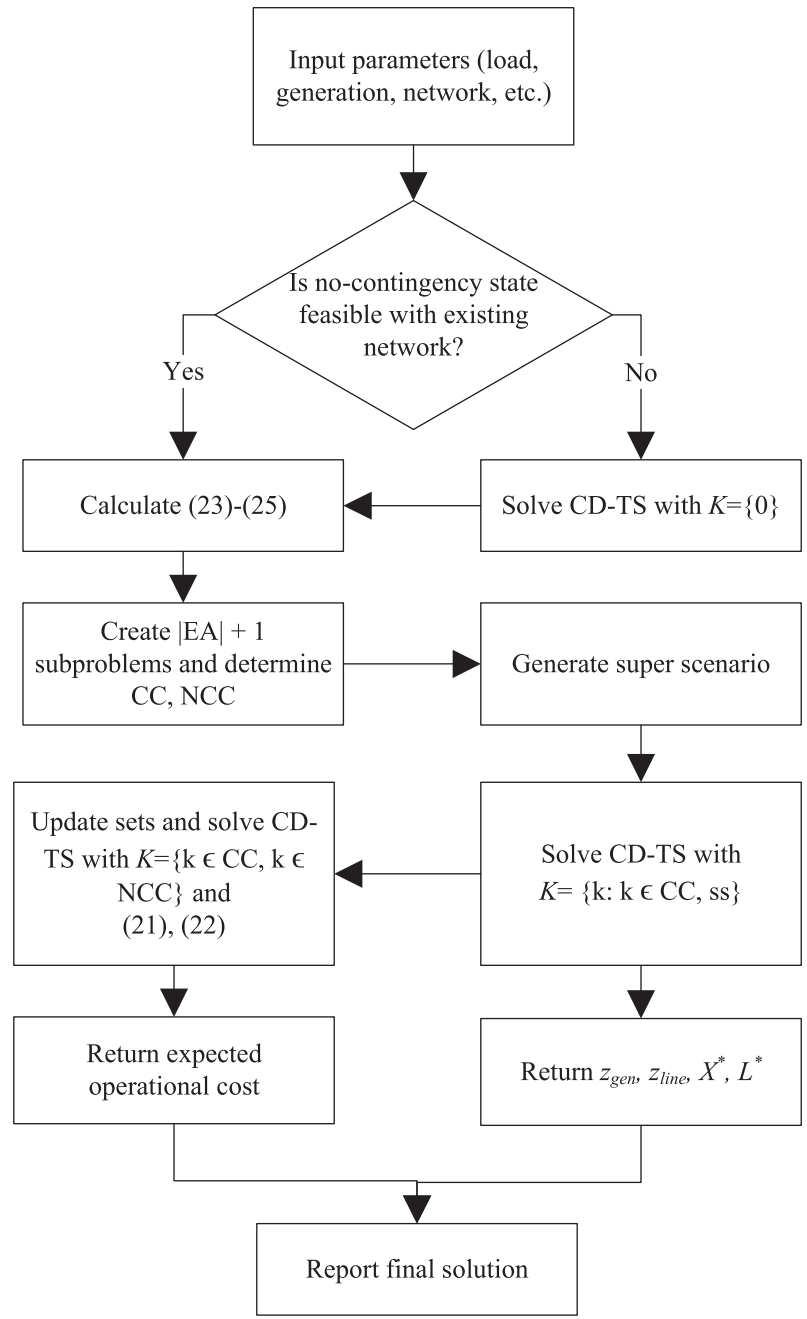

Fig. 1. Flow chart of the proposed scenario reduction based solution methodology (SRB).

Return the expected operational cost and report the final solution by combining with the output obtained at the end of Step 4. The flow chart of the solution methodology is presented in Fig. 1.

\section{Computational study}

In this section, we discuss the value of considering expected operational cost and effect of contingency-dependent TS. The model (CDTS) and the scenario reduction based solution methodology (SRB) is applied to the IEEE 24-bus and IEEE 118-bus power systems. The results of SRB are also presented for the Turkish power system. Experiments are performed on a Linux environment with a 4xAMD Opteron Interlagos 16C 6282SE 2.6G 16M 6400MT server with 96 GB RAM. Solution approach is implemented in Java Platform and results are obtained in Cplex 12.6.0 in parallel mode using up to 32 threads.

\subsection{IEEE 24-Bus power system}

IEEE 24-bus power system includes 24 nodes, 32 generation plants and 35 corridors for building transmission lines. The parameters of the existing generation units and transmission lines are given in [40]. We use the same configuration of the network and expansion alternatives for the generation units and lines presented in [39].

In this section, we analyze six cases with different levels of switching: no-switch case and the cases with the number of lines that can be switched is restricted from 1 to 5 . We add the following constraints 
to CD-TS for this restriction:

$L_{a} \leqslant S_{a}^{k}+z_{a} \quad \forall a \in A, k \in K$

$\sum_{a \in A} z_{a} \leqslant \tau$

$z_{a}$ is a binary variable which takes the value 1 if line $a$ is switched in any scenario $k$ and 0 o.w. $\tau$ is the number of transmission lines that can be switched. For the following analyses, we use Eqs. (23)-(25) to calculate the probability of scenarios.

We first emphasize the benefits of two-stage stochastic programming approach. Table 1 presents the results of the proposed CD-TS model and expected value of perfect information (EVPI) for the six cases defined above. The difference between the optimal values of CD-TS and EVPI is referred to as the maximum value that the system operator would pay for acquiring additional information for the uncertainty. In our problem setting, since the outage of the lines are considered as the source of uncertainty, the system operator is considered to be willing to pay almost $\$ 10 \mathrm{M}$ to handle this uncertainty.

We then discuss the benefits of the proposed transmission switching concept by comparing the solutions of CD-TS with the solutions when preventive security constrained TS is applied. For preventive security constrained TS concept, we guarantee that the network topology remains the same for all contingencies with the following equation and CD-TS with Eq. (30) is referred as PSC-TS.

$L_{a}=S_{a}^{k}+z_{a} \quad \forall a \in A, \quad k \in K$

Fig. 2 compares the optimal solution values of CD-TS and PSC-TS for the six switching possibilities. By allowing different network topologies for each contingency, the optimal solution values are reduced up to $1.92 \%$ and the highest improvement is obtained when at most 5 lines are allowed to be switched $(\tau=5)$. We note that, the optimal solution values of CD-TS always decreases as $\tau$ increases. However, the optimum solution values of PSC-TS are the same for the cases where the number of switchable lines, $\tau$, are larger than or equal to 2 . Hence, we conclude that, the number of switchable lines for PSC-TS can be insignificant after some point, although it is valuable for CD-TS.

Fig. 2 also shows the optimal solution values of the model for the same instances when reliability is not considered to evaluate the effect of reliability in the problem. For this analysis, we eliminate all the scenarios associated with the contingency states and the constraints related to these scenarios, in other words, the contingency set $K$ includes only the scenario for the no-contingency state, i.e. $K=\{0\}$. In order to be consistent with the reliability considered solutions (CD-TS and PSC-TS), we update the probability of no-contingency scenario for the case when reliability is not considered. For this case, the probability of the scenario $k=0$ is equal to the sum of all scenarios, $\left(p^{0}=\sum_{k \in K} p^{k}\right)$. As can be observed in Fig. 2, for all switching cases the optimal solutions without having any reliability consideration are significantly less than the optimal solutions of the reliability considered solutions. The difference between the solutions of the case without reliability and CD-TS can be interpreted as the cost of incorporating reliability into the power system, which costs about \$15 M. Note that, when the preventive security constrained TS approach is applied, the required investments will be more costly than the required investment cost with contingency-dependent TS approach. We also note that, EVPI and without reliability solutions are presented to evaluate different concepts. While the first one discusses the value of perfect information, the latter one analyses the effect of considering reliability in an optimal decision.

Table 2 details the solutions of CD-TS and PSC-TS and reports the installed and switched lines for the six cases. As expected, when $\tau=0$, the installed lines are the same for both CD-TS and PSC-TS as they reduce to the same problem without switching option. For the cases with $\tau=1$ and $\tau=2$, although the number of installed lines are the same, the switched lines are different. Hence, the key difference between the optimal solution values is due to their expected operational costs. Thus, by only using different network topologies for each scenario, the expected operational cost and therefore, the total system cost can be decreased. When contingency-dependent TS concept is used in the power system, not only switched lines but also expansion plans are affected. In all cases except for $\tau=0$ and $\tau=1$, at least one of the new transmission lines is different for the two switching approaches. Moreover, as in cases with $\tau=4$ and $\tau=5$, one less transmission line is built when contingency-dependent TS concept is applied. We again emphasize that, the solutions with preventive security constrained TS concept are the same when $\tau \geq 2$. Thus, the number of switched lines happens to be 2 in the optimal solution of PSC-TS even though switching more than 2 lines is allowed.

Table 2 also presents the value of transmission switching. For the IEEE 24-bus power system, we compare the solutions for the six cases $(0 \leq \tau \leq 5)$ as the solutions with $\tau>5$ remain almost the same for the CD-TS. Therefore, to discuss the value of TS, we compare the solutions obtained with $\tau=0$ and $\tau=5$, where they correspond to without switching and with switching cases, respectively. Even for this small dataset, the value of switching is valuable and a $3.87 \%$ decrease in the total system cost is achieved by only allowing switching in the network for CD-TS. Moreover, when switching is not used, 2 more lines (i.e. $(9,12)$ and $(16,17)$ should be built to maintain the required reliability level. Thus, by allowing TS in the system, expansion plans can be affected beside decreasing the total system cost.

Fig. 3(a) and (b) present the optimal solutions of the transmission switching concepts compared to the case where only operation cost for no-contingency scenario, $k=0$, is included to the objective function of CD-TS and PSC-TS, respectively. We observe that the solutions that only consider the operational cost of no-contingency scenario underestimate the total expected cost and especially for small $\tau$ values, the difference between the solutions are significant and up to $\$ 2.74 \mathrm{M}$ cost is underestimated for this example if the outcomes during the contingency states are ignored. We also note the underestimated monetary value might be higher than this one for the power systems with more flexible generators or renewable generator with highly variable outputs.

Table 3 details the solutions of Fig. 3(a) and presents the installed and switched lines for the proposed switching concept. In the case with $\tau=2$, as all the installed and switched lines are the same, the cost difference is due to not considering operation costs during the contingency states. However, as in other cases, including expected operational cost to the problem not only affect the optimal solution values, but also change the expansion plans. For the cases with $\tau=1$ and $\tau=3$, one more transmission line is installed when the expected operational cost term is included to the objective function and the key difference between the solutions of these instances is due to the change in $z_{\text {line }}$. In other cases with $\tau \geq 4$, although the number of installed lines are the same, at least one of the switched lines are different from each other, which is also one of the reasons for the difference between the optimal solution values as the generation outputs are different from each other. Therefore, considering probabilistic realization of outages and defining transmission switching as recourse actions affect the planning decisions, network topologies and cost of the expansion plans.

Table 4 compares solution times of the proposed method (SRB) with the solution times of proposed CD-TS model. In all the cases considered

Table 1

Value of two-stage stochastic programming on IEEE 24-bus power system.

\begin{tabular}{llll}
\hline$\tau$ & CD-TS (M\$) & EVPI (M\$) & Difference (\%) \\
\hline 0 & 153.74 & 141.18 & 8.90 \\
1 & 151.63 & 139.08 & 9.02 \\
2 & 149.61 & 138.95 & 7.67 \\
3 & 148.93 & 138.67 & 7.39 \\
4 & 147.92 & 138.63 & 6.70 \\
5 & 147.79 & 138.53 & 6.68 \\
\hline
\end{tabular}




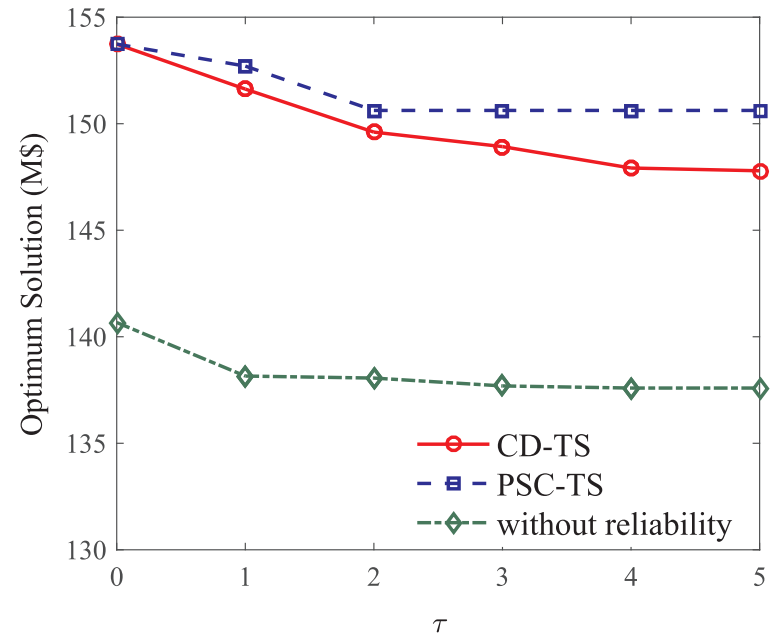

Fig. 2. Optimal solutions of CD-TS, PSC-TS and without reliability.

Table 2

Installed and switched lines in the solutions of CD-TS and PSC-TS on IEEE 24bus power system.

\begin{tabular}{|c|c|c|c|}
\hline$\tau$ & & Installed Lines & Switched Lines \\
\hline 0 & $\begin{array}{l}\text { CD-TS } \\
\text { PSC-TS }\end{array}$ & $\begin{array}{c}(3,24)(7,8)(9,12)(15,21) \\
(15,24)(16,17)(20,23) \\
(3,24)(7,8)(9,12)(15,21) \\
(15,24)(16,17)(20,23)\end{array}$ & \\
\hline 1 & $\begin{array}{l}\mathrm{CD}-\mathrm{TS} \\
\overline{\mathrm{PS}} \overline{\mathrm{C}}-\overline{\mathrm{T}} \overline{\mathrm{S}}\end{array}$ & $\begin{array}{c}(3,24)(7,8)(14,16)(15,21) \\
(15,24)(16,17)(20,23) \\
(\overline{3}, \overline{24}) \overline{(7,8)}(1 \overline{4}, \overline{16} \overline{-} \overline{(15,21)} \\
\quad(15,24)(16,17)(20,23)\end{array}$ & $\begin{array}{c}\frac{(6,10)}{(1, \overline{2})} \\
-------\end{array}$ \\
\hline 2 & $\begin{array}{l}\text { CD-TS } \\
\overline{\mathrm{PS}} \overline{\mathrm{C}}-\overline{\mathrm{T}} \overline{\mathrm{S}}\end{array}$ & $\begin{array}{c}(3,24)(7,8)(15,21) \\
(15,24)(16,17)(20,23) \\
--(3,24)-(7,8)(14,16) \\
(15,21)(15,24)(20,23)\end{array}$ & $\begin{array}{r}(6,10)(9,11) \\
--\overline{(15}, \overline{16} \overline{)} \overline{(17}, \overline{1}, \overline{8})\end{array}$ \\
\hline 3 & $\begin{array}{l}\text { CD-TS } \\
\overline{\mathrm{PS}} \overline{\mathrm{S}} \overline{\mathrm{C}}-\overline{\mathrm{T}} \overline{\mathrm{S}}\end{array}$ & $\begin{array}{c}(3,24)(7,8)(15,21) \\
(15,24)(16,17)(20,23) \\
---(\overline{3}, 24)(7,8)(14,16) \\
(15,21)(15,24)(20,23)\end{array}$ & $\begin{array}{c}(2,4)(6,10)(9,11) \\
--\overline{(15}, \overline{1} \overline{6}) \overline{(17}, \overline{1} \overline{8})\end{array}$ \\
\hline 4 & $\begin{array}{l}\text { CD-TS } \\
\overline{\mathrm{PS}} \overline{\mathrm{S}} \overline{\mathrm{C}}-\overline{\mathrm{T}} \overline{\mathrm{S}}\end{array}$ & $\begin{array}{c}(3,24)(7,8)(15,21) \\
(15,24)(20,23) \\
--\overline{(3,24)} \overline{(7,8)}(1 \overline{4}, 16 \overline{)}- \\
(15,21)(15,24)(20,23)\end{array}$ & $\begin{array}{r}(6,10)(10,11) \\
--\frac{(15,16)}{(15, \overline{1}} \overline{16} \frac{(17,18)}{(17, \overline{18})}---\end{array}$ \\
\hline 5 & $\begin{array}{l}\mathrm{CD}-\mathrm{TS} \\
\overline{\mathrm{PS}} \overline{\mathrm{S}}-\overline{\mathrm{T}} \overline{\mathrm{S}}\end{array}$ & $\begin{array}{c}(3,24)(7,8)(15,21) \\
(15,24)(20,23) \\
---\overline{(3,24)} \overline{(7,8)}(1 \overline{4}, \overline{16})-- \\
(15,21)(15,24)(20,23)\end{array}$ & 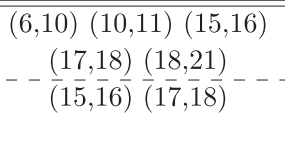 \\
\hline
\end{tabular}

in this study, SRB finds the optimal solutions in significantly less solution time and up to $94.07 \%$ improvement is achieved for the case with $\tau=5$, when the SRB is applied and on the average the improvement in the solution times is $78.27 \%$. Thus, it is a prominent solution methodology to overcome the computational complexity of reliability constrained problems that can lead to memory problems. In the following sections, we test the performance of proposed methodology on larger datasets.

\subsection{IEEE 118-bus power system}

IEEE 118-bus power system includes 118 buses, 19 generation plants and 186 transmission lines $[28,41]$. In the original network, the total installed capacity and total demand are $5859 \mathrm{MW}$ and $4519 \mathrm{MW}$, respectively. As the operation costs of generation units are relatively low compared to today's values provided in the next section for a reallife case, we multiply the operation costs of generation units by 2.5 . We also reduce the capacities of the transmission lines by $20 \%$ in order to increase the congestion and observe the effect of TS on the grid. Forced outage rates (FOR) of transmission lines, $\sigma_{a}$, are set to 0.005 for all the lines.

Table 5 presents the results of our proposed CD-TS model and SRB methodology on this power system for two cases: (A) switching is allowed only on the new lines and (B) switching is allowed on all the lines. For the CD-TS model, we provide a warm-start solution using our SRB methodology with a starting value of $\$ 47.23 \mathrm{M}$. But, the solution is not improved for the next $12 \mathrm{~h}$ and the reported gaps by the solver at the end of $12 \mathrm{~h}$ time limit, are $16.79 \%$ and $23.92 \%$ for the cases A and $\mathrm{B}$, respectively.

For this example, Step 2 of SRB finds 16 and 9 critical contingencies (or scenarios) for the cases A and B, respectively. Thus, we also verify our motivation for the SRB methodology as most of the contingencies do not affect power system reliability. As we reduce the number of scenarios in the system, we find the solutions for these instances using SRB in significantly shorter solution times. We obtain solutions in less than $1 \mathrm{~h}$ for case A, and less than $6 \mathrm{~h}$ for case B. We also emphasize that in both cases, the solutions obtained from SRB methodology is less than the best integer solution obtained with CD-TS model within $12 \mathrm{~h}$. Hence, efficiency of the SRB methodology is more obvious for this large data set. We also emphasize the benefits of two-stage stochastic programming approach that includes the expected value of operational costs in the objective function. By solving several single-scenario problems on the IEEE 118-bus power system and taking the expected value of the solutions of the single scenario problems, we calculate the expected value of perfect information (EVPI) for both cases. The differences between the solutions of SRB and EVPI are $15.92 \%$ and $15.78 \%$ for cases A and B, respectively, which is the maximum value that system operator is considered to be willing to pay to acquire additional information for the outage of transmission lines.

Table 6 details the solutions of SRB methodology for the two cases to analyze the value of transmission switching. As switching is allowed on all the lines, a $10.13 \%$ improvement is obtained and not only investment cost, but also expected operational cost decreases with incorporating switching option for all lines. Moreover, switching lines can affect the investment plans as one less transmission line is built in case $\mathrm{B}$, which costs approximately $\$ 2 \mathrm{M}$.

\subsection{Turkish power system}

Turkish transmission network is comprised of $380-\mathrm{kV}, 220-\mathrm{kV}, 154-$ $\mathrm{kV}$ and $66-\mathrm{kV}$ voltage levels. $380-\mathrm{kV}$ transmission network is considered as the Turkish main transmission system [42] and this paper analyzes this backbone network in terms of N-1 reliability criterion.

Fig. 4 presents the $380-\mathrm{kV}$ transmission network and the substations. Table 7 summarizes the transmission network, demand and generation data for 2016 [43]. As demands of the buses are not available, for this analysis, we calculate the demand of each node based on the profiles provided in [44]. The characteristics of the overhead transmission lines and generation technologies are presented in Table 8. Underground cables are also built in residential areas.

We apply a similar methodology referred to as power island model [45] to reduce the size of the data that we used in our analysis. We assume a generation unit dispatches power to its closest substation and demand nodes are fed from their closest substations. In this power island model, we assign all the demand nodes and generation units to their closest substations. We aggregate demand values and generation capacities to 118 substations and these substations are considered as nodes (buses) in the model. Thus, at the end of this procedure, we obtain a simplified Turkish power system with 118 buses and 245 existing transmission lines. The schematic representation of the power island model is shown in Fig. 5. 


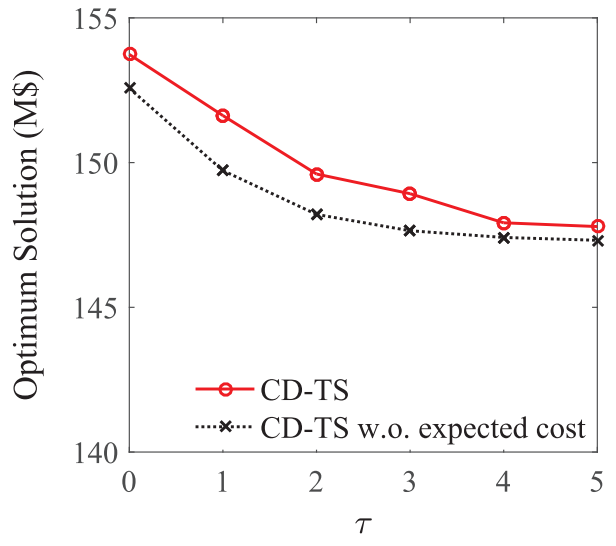

(a)

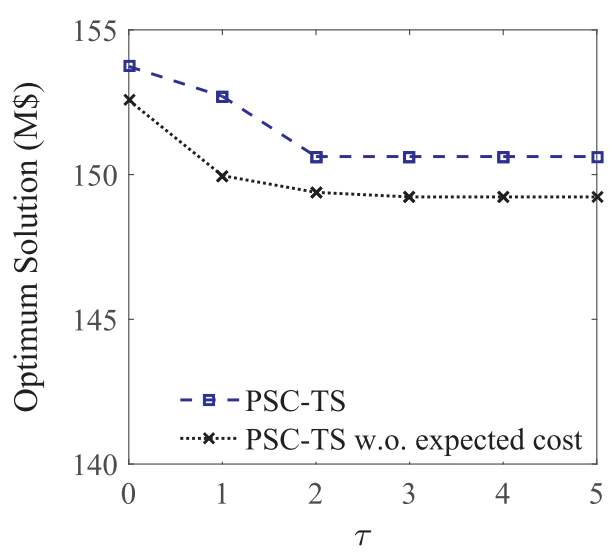

(b)

Fig. 3. Value of adding expected operational cost to (a) CD-TS (b) PSC-TS.

Candidate transmission lines and locations of generation units are in accordance with future expansion plans [46]. The number of candidate generation units is 696 that can be built at 103 buses. The parameters of candidate generation units for each technology is presented in Table 9 and we estimate capital and operational costs of them by utilizing the data in [47]. The parameters are also the same for the existing plants. The life time of new power plants is taken as 30 years and the discount rate that includes inflation is $5 \%$. 3xCardinal, 3xPheasant and underground cables are considered for the new lines. All corridors are considered for expansion and at most two lines can be built from each type on the same corridor. The investment costs of these transmission lines are estimated as $\$ 1.7 \mathrm{M} / \mathrm{km}$ for $3 \times$ Cardinal type and $\$ 1.9 \mathrm{M} / \mathrm{km}$ for
Table 4

Solution times of the model and the solution methodology.

\begin{tabular}{llll}
\hline$\tau$ & CD-TS (h) & SRB (h) & Improvement (\%) \\
\hline 0 & 0.13 & 0.02 & 87.45 \\
1 & 1.23 & 0.24 & 80.49 \\
2 & 4.89 & 2.67 & 45.40 \\
3 & 18.97 & 2.53 & 86.66 \\
4 & 7.04 & 1.72 & 75.57 \\
5 & 11.30 & 0.67 & 94.07 \\
Average & 7.26 & 1.31 & 78.27 \\
\hline
\end{tabular}

Table 3

Installed and switched lines in the solutions of CD-TS and PSC-TS on IEEE 24-bus power system.

\begin{tabular}{|c|c|c|c|}
\hline$\tau$ & & Installed Lines & Switched Lines \\
\hline 0 & $\begin{array}{c}\text { CD-TS } \\
\text { CD-TS w.o. } \exp \text { cost }\end{array}$ & $\begin{array}{c}(3,24)(7,8)(9,12)(15,21) \\
(15,24)(16,17)(20,23) \\
(3,24)(7,8)(9,12)(15,21) \\
(15,24)(16,17)(20,23)\end{array}$ & \\
\hline \multirow[t]{2}{*}{1} & CD-TS & $\begin{array}{c}(3,24)(7,8)(14,16)(15,21) \\
(15,24)(16,17)(20,23)\end{array}$ & $(6,10)$ \\
\hline & $\overline{\mathrm{C}} \overline{\mathrm{D}}-\overline{\mathrm{TS}}{ }^{-}$w.o. exp cost & $\begin{array}{c}(3,9)(7,8)(14,16) \\
(15,21)(16,17)(20,23)\end{array}$ & $\overline{(6, \overline{10})}$ \\
\hline \multirow[t]{2}{*}{2} & CD-TS & $\begin{array}{c}(3,24)(7,8)(15,21) \\
(15,24)(16,17)(20,23)\end{array}$ & $(6,10)(9,11)$ \\
\hline & $\overline{\mathrm{C}} \overline{\mathrm{D}}-\overline{\mathrm{TS}}$ w.o. exp cost & $\begin{array}{c}(3,24)(7,8)(15,21) \\
(15,24)(16,17)(20,23)\end{array}$ & $(\overline{6}, \overline{1} 0) \overline{(9,1 \overline{1}})$ \\
\hline \multirow[t]{2}{*}{3} & CD-TS & $\begin{array}{c}(3,24)(7,8)(15,21) \\
(15,24)(16,17)(20,23)\end{array}$ & $(2,4)(6,10)(9,11)$ \\
\hline & $\overline{\mathrm{C}} \overline{\mathrm{D}}-\overline{\mathrm{TS}}$ w.o. exp cost & $\begin{array}{c}(3,24)(7,8)(15,21) \\
(15,24)(20,23)\end{array}$ & $(\overline{6}, 10)(9,1 \overline{1})(\overline{1} 5,1 \overline{6})$ \\
\hline \multirow[t]{2}{*}{4} & CD-TS & $\begin{array}{c}(3,24)(7,8)(15,21) \\
(15,24)(20,23)\end{array}$ & $\begin{array}{c}(6,10)(10,11) \\
(15,16)(17,18)\end{array}$ \\
\hline & $\overline{\mathrm{C}} \overline{\mathrm{D}}-\overline{\mathrm{TS}}$ w.o. exp cost & $\begin{array}{c}(3,24)(\overline{7}, \overline{8})(\overline{1} 5,21) \\
(15,24)(20,23)\end{array}$ & $\begin{array}{l}(6,10)(10,11) \\
(15,16)(18,20)\end{array}$ \\
\hline \multirow[t]{2}{*}{5} & CD-TS & $\begin{array}{c}(3,24)(7,8)(15,21) \\
(15,24)(20,23)\end{array}$ & $\begin{array}{c}(6,10)(10,11)(15,16) \\
(17,18)(18,21)\end{array}$ \\
\hline & $\overline{\mathrm{C}} \overline{\mathrm{D}}-\overline{\mathrm{TS}}$ w.o. exp cost & $\begin{array}{c}(3,24)(7,8)(15,21) \\
(15,24)(20,23)\end{array}$ & $\begin{array}{l}(1,2)(6,10)(10,11) \\
(15,16)(18,21)\end{array}$ \\
\hline
\end{tabular}


Table 5

Results for CD-TS and SRB on IEEE 118-bus power system.

\begin{tabular}{llll}
\hline & & $\begin{array}{l}\text { Case A (New lines } \\
\text { switchable) }\end{array}$ & $\begin{array}{l}\text { Case B (All lines } \\
\text { switchable) }\end{array}$ \\
\hline \multirow{2}{*}{ CD-TS } & Best solution (M\$) & 47.23 & 47.23 \\
& Solution time (h) & $>12$ & $>12$ \\
& Gap (\%) & 16.79 & 23.92 \\
SRB & Best solution (M\$) & 41.65 & 37.43 \\
& Solution time (h) & 0.78 & 5.51 \\
\hline
\end{tabular}

Table 6

Results for the IEEE-118 bus power system.

\begin{tabular}{|c|c|c|}
\hline & $\begin{array}{l}\text { Case A (New lines } \\
\text { switchable) }\end{array}$ & $\begin{array}{l}\text { Case B (All lines } \\
\text { switchable) }\end{array}$ \\
\hline$z_{\text {line }}(\mathrm{M} \$)$ & 11.40 & 9.45 \\
\hline \# of new lines & 12 & 11 \\
\hline Expected op. cost (M\$) & 30.25 & 27.98 \\
\hline
\end{tabular}

3xPheasant type [48]. Life time of transmission lines is taken as 50 years with the same interest rate. We calculate the probability of each contingency based on the system fault index of transmission system [49]. The details of the data and related analyses can be shared upon contacting with authors.

The proposed SRB solution methodology is applied to $380-\mathrm{kV}$ simplified Turkish power system for the same two cases used in the previous section and in case $\mathrm{A}$, we only allow switching on the new lines and in case B, we allow switching on all the lines. Step 2 of SRB identifies the redundant contingencies and at this step after temporarily removing the redundant ones, 29 and 12 of them are considered as critical contingencies and added to the set CC for cases A and B, respectively. Thus, in any cases, the current system does not satisfy N-1 reliability criterion. As seen in Fig. 4, there are radial $380-\mathrm{kV}$ transmission elements which are also counted in this analysis. However, these radial lines can be removed from the contingency set as in [28]. Table 10 summarizes the results of the solution method for the two cases. When switching is allowed on the new lines (case A), 14 transmission lines are installed with a total investment cost of $\$ 82.38 \mathrm{M}$ and when switching is allowed on all the lines (case B), 11 transmission lines are installed to satisfy the N-1 reliability criterion and the total investment cost is $\$ 79.85 \mathrm{M}$. Hence, we observe that using contingencydependent TS for all the lines decreases the number of lines that should be installed by 3 and cost by $\$ 14.78 \mathrm{M}$.

The difference in the solutions are also shown in Fig. 6 for the Kocaeli-Istanbul region. Fig. 6 presents the substations, existing lines between these substations (thin lines) and installed lines (bold lines) for the cases A and B. Fig. 6(b) shows four of the 11 installed lines for case B. When the switchable lines are restricted with the new lines, two more lines are installed in the same region which is presented in
Table 7

Summary of the Turkish power system data.

\begin{tabular}{ll} 
\# of nodes (buses) & 970 \\
\# of transmission lines & 245 \\
\# of substations & 118 \\
\# of generation units & 1244 \\
Total peak demand & $44,734 \mathrm{MW}$ \\
Total generation capacity & $77,737 \mathrm{MW}$ \\
\hline
\end{tabular}

Table 8

Characteristics of Turkish power system data.

\begin{tabular}{|c|c|c|c|c|c|}
\hline \multicolumn{3}{|c|}{ Transmission Lines } & \multicolumn{3}{|c|}{ Generation Units } \\
\hline Type & $\begin{array}{l}\text { Cap. } \\
\text { (MW) }\end{array}$ & $\begin{array}{l}\text { Reactance } \\
\text { (ohm/km) }\end{array}$ & Technology & $\#$ & $\begin{array}{l}\text { Distribution of } \\
\text { capacity (\%) }\end{array}$ \\
\hline 2xRail & 500 & 0.3190 & Thermal & 452 & 56.59 \\
\hline 2xCard. & 500 & 0.3168 & Hydro & 605 & 34.32 \\
\hline 3xCard. & 750 & 0.2621 & Wind & 153 & 7.39 \\
\hline \multirow[t]{2}{*}{ 3xPhea. } & 1000 & 0.2559 & Geoth. & 32 & 1.06 \\
\hline & & & Solar & 2 & 0.02 \\
\hline
\end{tabular}

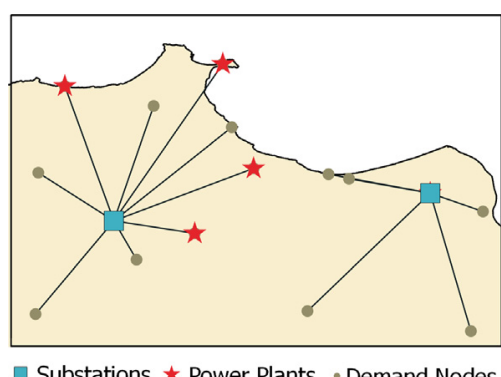

Fig. 5. Power island model.

Fig. 6(a). Although in the current power system, switching transmission lines can raise different problems, transmission switching in expansion planning is worth to discuss [32] as the investment plans and estimated operational costs of generation can be significantly affected.

\section{Extensions and discussions}

In this section, we discuss the extensions of the focused problem explained in Section 1. The problem setting can be easily modified for the possible extensions that could be multi-stage expansion planning, demand uncertainty or renewable generation uncertainty and in the following sections, we will analyze the multi-stage expansion planning and demand uncertainty cases among the possible ones. We first explain the modifications to the proposed CD-TS model and proposed SRB solution approach. We then discuss the results obtained with the modified versions of the CD-TS and SRB for the IEEE 24-bus power system and

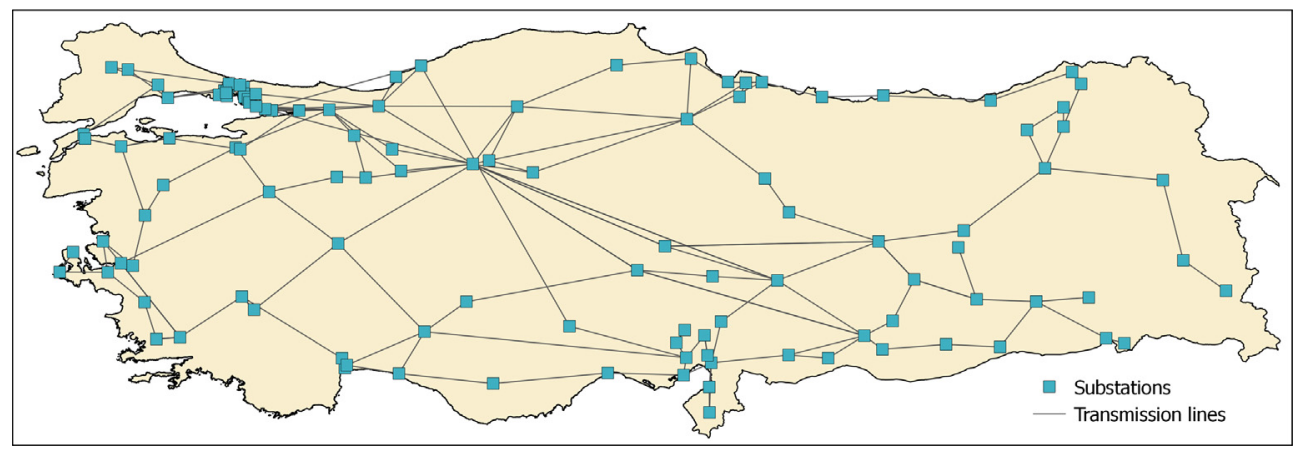

Fig. 4. Substations and lines on $380-\mathrm{kV}$ transmission network in Turkey. 
Table 9

Characteristics of the generation technologies.

\begin{tabular}{lllll}
\hline Type & $\begin{array}{l}\text { Capacity } \\
\text { (MW) }\end{array}$ & $\begin{array}{l}\text { Capital cost }(\mathrm{M} \\
\text { \$/MW-year. }\end{array}$ & $\begin{array}{l}\text { O\&M cost } \\
(\$ / M W h)\end{array}$ & $\begin{array}{l}\text { Capacity factor } \\
(\%)\end{array}$ \\
\hline Thermal & 500 & 0.08 & 4.28 & 80.00 \\
Hydro & 350 & 0.15 & 6.85 & 29.00 \\
Wind & 150 & 0.10 & 4.57 & 28.00 \\
Geo. & 100 & 0.16 & 5.71 & 78.00 \\
Solar & 100 & 0.11 & 1.90 & 17.00 \\
Nuclear & 2000 & 0.29 & 17.88 & 85.00 \\
\hline
\end{tabular}

Table 10

Results for the 380-kV Turkish transmission network.

\begin{tabular}{lll}
\hline & $\begin{array}{l}\text { Case A (New lines } \\
\text { switchable) }\end{array}$ & $\begin{array}{l}\text { Case B (All lines } \\
\text { switchable) }\end{array}$ \\
\hline \# new lines & 14 & 11 \\
$z_{\text {line }}(\mathrm{M} \$)$ & 82.38 & 79.85 \\
Expected op. cost $(\mathrm{M} \$)$ & 933.47 & 918.69 \\
\hline
\end{tabular}

Turkish transmission network for the extensions.

\subsection{Multi-stage expansion planning}

The proposed CD-TS model is easily extendable for multi-stage expansion planning problem with an additional dimension $t$ to represent the decisions in year $t$. As the demand is exogenously given to the model, we also add the time dimension $t$ to $l_{i}$, such that $l_{i}^{t}$ is the demand of node $i$ in year $t$. Each constraint set of the model CD-TS is reproduced for each year $t \in T$ where $T$ is the planning horizon. We also need to add the following constraints to the model:

$L_{a}^{t-1} \leqslant L_{a}^{t} \quad \forall a \in A, \quad t \in T$

$X_{i g}^{t-1} \leqslant X_{i g}^{t} \quad \forall i \in B, \quad g \in G, \quad t \in T$

Eqs. (31) and (32) are the time coupling equations. Eq. (31) guarantees that a new line built in year $t-1$ can be used in year $t$. Similarly, Eq. (32) guarantees that a new generation unit built in year $t-1$ can also be used in year $t$. We also note that the existing lines are added to the new version of the model by replacing Eq. (10) with the following one:

$L_{a}^{0}=1 \quad \forall a \in E A$

The proposed solution methodology can be extended for the multistage expansion planning by generating critical contingency $(C C)$ and non-critical contingency (NCC) lists for each time period. First, we decompose the multi-period problem into a set of single period problems as many as the number of expansion periods, $|T|$, and apply the steps of the SRB for the first subproblem (for $t=1$ ) and get the new investments. Then we fix these new investments and apply the steps of

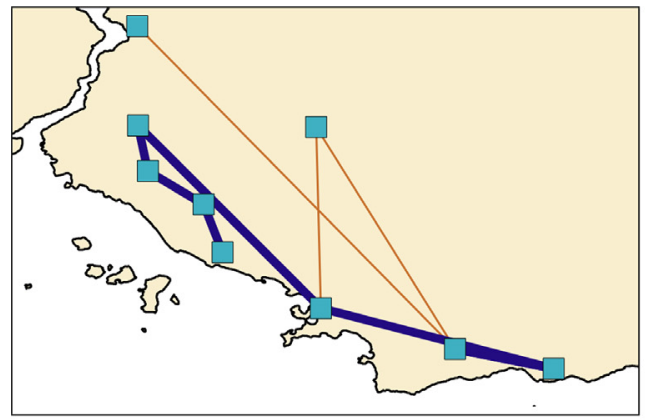

(a)
Table 11

Results of CD-TS and SRB on IEEE 24-bus power system for multi stage expansion.

\begin{tabular}{|c|c|c|c|}
\hline & & $\begin{array}{l}\text { Case A (New lines } \\
\text { switchable) }\end{array}$ & $\begin{array}{l}\text { Case B (All lines } \\
\text { switchable) }\end{array}$ \\
\hline \multirow[t]{3}{*}{ CD-TS } & Best solution (M\$) & 718.68 & 926.98 \\
\hline & Solution time (h) & 0.39 & $>12$ \\
\hline & Gap (\%) & - & 23.73 \\
\hline \multirow[t]{2}{*}{ SRB } & Best solution (M\$) & 751.04 & 730.18 \\
\hline & Solution time (h) & 0.01 & 0.01 \\
\hline
\end{tabular}

the solution methodology for the second subproblem (for $t=2$ ) and get the optimal solution. After solving all single-period problems iteratively by the proposed SRB method in Section 2.2, we combine all the solutions for each subproblem and get the solution for the original multistage expansion planning problem.

Table 11 presents the solutions of the modified versions of the CDTS model and SRB method for the IEEE 24-bus power system for the same cases discussed in Sections 3.2 and 3.3. Annual demand growth rate is assumed to be $3 \%$ for the next 4 years and capacities of transmission lines are reduced by $40 \%$. The modified version of CD-TS model finds the optimal solution of case A (i.e. only new lines are switchable) within $24 \mathrm{~min}$. However, when all lines are switchable, the optimality of the problem is not verified within $12 \mathrm{~h}$ time limit and the solver reports $23.72 \%$ gap at the end of the time limit. For the same cases, although the modified version of SRB cannot find the optimal solution of case A, the solution obtained with the SRB for case B is significantly lower than the solution obtained with the CD-TS at the end of time limit. As the solution times of the SRB method is significantly less than the solution times of the CD-TS, we conclude that the proposed solution approach is still a promising method to decrease computational complexity of the problem.

We also apply the modified version SRB solution method to the 380$\mathrm{kV}$ Turkish transmission network. Estimated annual growth rate is given as $4.87 \%$ (average) for the next 4 years [50]. Table 12 presents the results of the SRB method for the same cases for the Turkish transmission network. At the end of planning horizon, as $21 \%$ demand increase is estimated, a new generator is built at the same place in both cases. However, similar to previous discussions in Section 3.3, transmission switching decreases the expected operational cost in the system. Moreover, as switching lines affect the expansion plans, the number of lines in case B is also less than the number of lines in Case A. We note that, we use the estimated annual growth rate to increase the demand. However, more detailed analyses can be conducted by considering load blocks within each year. Demand in each load block can be estimated using a similar technique described in [51] to consider the correlations between the load blocks.

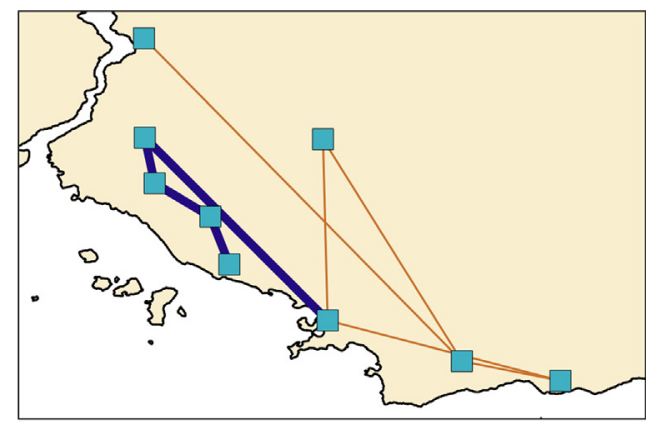

(b)

Fig. 6. (a) Installed lines (bold lines) (a) when switching is allowed only on the new lines (b) when switching is allowed on all the lines. 
Table 12

Results for the $380-\mathrm{kV}$ Turkish transmission network for multi stage expansion.

\begin{tabular}{lll}
\hline & $\begin{array}{l}\text { Case A (New lines } \\
\text { switchable) }\end{array}$ & $\begin{array}{l}\text { Case B (All lines } \\
\text { switchable) }\end{array}$ \\
\hline \# new generator & 1 & 1 \\
\# new lines & 25 & 19 \\
$z_{\text {line }}(\mathrm{M} \$)$ & 120.86 & 98.41 \\
Expected op. cost (M\$) & 5255.61 & 5220.01 \\
\hline
\end{tabular}

\subsection{Demand uncertainty}

In Section 2, the proposed two-stage stochastic model includes the probabilistic realization of outages in transmission lines. Our model can easily be extended and other uncertainties can be incorporated into the model at an expense of increased computation time and memory. In this subsection, we discuss the effect of including demand uncertainty to the problem and the modifications required to handle these uncertainties in the proposed model and solution methodology. Different demand levels are considered to take into account demand uncertainty.

For this extension, we keep first stage decision variables, $X_{i g}$ and $L_{a}$ as in CD-TS. Each decision variables in the second stage such as power flow and generation amount have a new dimension $w$ to represent the demand levels. We also change the parameter $l_{i}$ with $l_{i}^{w}$ to represent load in bus $i$ in demand level $w$. Each set of Eqs. (2)-(9) and Eqs. (11) and (12) should be satisfied for each demand level $w \in W$ where $W$ is the set of demand levels. We then modify the objective function to incorporate demand uncertainty as we assume independent and identically distributed outages of transmission lines and demand levels. In Eq. (33), $\rho^{w}$ represents the probability of demand level $w$.

$\min z_{\text {gen }}+z_{\text {line }}+\sum_{w \in W} \rho^{w} \sum_{k \in K} z_{o m}^{k w}$

In the SRB approach, for handling different demand levels, we modify Step 3 and Step 4 of the methodology explained in Section 2. We now define $C C^{w}$ and $N C C^{w}$ for the critical and non-critical contingencies in demand level $w$ and generate a super scenario for each $w \in W,\left(s s^{w}\right)$. We then define $K^{w}$ for the contingency set of demand level $w$, i.e. $K^{w}=\left\{k: k \in C C^{w}\right.$ and $\left.s s^{w}\right\}$. We then solve the CD-TS for each demand level $w$ and for each scenario in the contingency set of the $w,\left(\forall w\right.$ and $\left.\forall k \in K^{w}\right)$.

Table 13 compares the results of the modified versions of CD-TS, PSC-TS and SRB methodology for the IEEE 24-bus power system for the two cases given above. In this analysis we consider three demand levels $\left(l_{i}^{1}=l_{i}, l_{i}^{2}=1.05 l_{i}\right.$ and $\left.l_{i}^{3}=1.1 l_{i}\right)$ as in [52] with equal probabilities. Similar to the results in Table 11, the modified version of CD-TS finds the optimal solution for case A within $25 \mathrm{~min}$, whereas it concludes with $8.37 \%$ gap at the end of $12 \mathrm{~h}$ time limit. In these instances, the modified version of SRB method finds the optimal solution of case A within $19 \mathrm{~min}$. Moreover, the solution obtained with the proposed method for case B is lower than the solution obtained with the CD-TS at the end of time limit.

Table 13 also discuss the benefits of the proposed switching concept. When switching is allowed on only new lines, the value of the switching is not remarkable. However, when switching is allowed on all the lines, despite the fact that the optimality of case B is not verified by the CDTS, we obtained a solution with $\$ 150.18 \mathrm{M}$ with the SRB method. Thus, the proposed switching concept leads to a $5.34 \%$ decrease in the total cost. We also note that, as the solution obtained from the SRB may not be optimal, the value of contingency-dependent switching may be higher than $5.34 \%$.

We then apply the SRB for the Turkish power system and Table 14 depicts the results. In the previous section, we provide the annual growth rate for the next 4 years. In this section, the demand in the year 2020 is considered as the medium demand level and a 10\% lower and higher than this average value is considered for the other demand
Table 13

Results of CD-TS, PSC-TS and SRB on IEEE 24-bus power system with demand uncertainty.

\begin{tabular}{llll}
\hline & & $\begin{array}{l}\text { Case A (New lines } \\
\text { switchable) }\end{array}$ & $\begin{array}{l}\text { Case B (All lines } \\
\text { switchable) }\end{array}$ \\
\hline \multirow{2}{*}{ CD-TS } & Best solution (M\$) & 159.71 & 162.09 \\
& Solution time (h) & 0.41 & $>12$ \\
& Gap (\%) & - & 8.37 \\
\multirow{2}{*}{ PSC-TS } & Best solution (M\$) & 159.78 & 158.65 \\
& Solution time (h) & 0.08 & 8.26 \\
SRB & Best solution (M\$) & 159.71 & 150.18 \\
& Solution time (h) & 0.31 & 2.01 \\
\hline
\end{tabular}

Table 14

Results for the 380-kV Turkish transmission network with demand uncertainty.

\begin{tabular}{lll}
\hline & $\begin{array}{l}\text { Case A (New lines } \\
\text { switchable) }\end{array}$ & $\begin{array}{l}\text { Case B (All lines } \\
\text { switchable) }\end{array}$ \\
\hline \# new generator & 1 & - \\
\# new lines & 31 & 29 \\
$z_{\text {line }}(\mathrm{M} \$)$ & 174.07 & 131.54 \\
Expected op. cost $(\mathrm{M} \$)$ & 3505.92 & 3555.89 \\
\hline
\end{tabular}

levels. When switching existing transmission lines are not allowed, a new generator and 31 new transmission lines are built in the optimal solution. However, when switching operations are allowed to all lines, the generator and two transmission lines are not required any more. On the other hand, expected operational cost is higher in case $B$ than the expected operation cost in case A. Hence, in case B, the expensive generators are utilized instead of building new generator and new transmission lines. We note here that, the modified SRB approach discussed above can be easily extended to the problems with different uncertainties such as solar, wind generators or market prices.

\section{Conclusion}

This paper presents a two-stage stochastic programming model for a N-1 reliability constrained generation and transmission expansion planning problem. Operational decisions such as status of transmission lines, generation amounts and power flow decisions are defined as recourse actions of the two-stage stochastic programming model for each contingency state and the model makes it possible to calculate the expected value of operational costs during the contingencies in a more accurate manner. A scenario reduction based solution methodology with a filtering technique is also proposed to overcome the computational complexity of the problem.

The model and the proposed solution approach are tested on the IEEE 24-bus and 118-bus power systems. We first show that considering the operational costs during the contingency states and changing the network topology for each contingency affect the expansion plans and overall costs of the expansion plans significantly. Our results demonstrate that the proposed contingency-dependent transmission switching concept can decrease the total system up to $10.13 \%$ and/or number of transmission lines. We also compare the solutions obtained with the model and proposed solution approach to discuss the computational efficiency of the solution method. For the IEEE 24-bus power system, the solution method finds the optimal solutions with significantly shorter solution times $(78.27 \%$ on the average). For the IEEE 118-bus power system, while the model cannot verify the optimality within the time limit, the solution method results with lower total system cost than the cost obtained with the model.

This paper also introduces a real-world data set for the $380-\mathrm{kV}$ Turkish transmission network. Using the proposed solution approach, we find expansion plans that satisfies the N-1 reliability criterion and show that allowing contingency-depending TS can reduce the total cost 
of the system. We also note that, the value of TS are expected to be more important for power systems that have flexible generator or renewable generator with highly variable outputs.

In this study, as customarily done in the literature, we plan for a target year, and all the discussions are demonstrated for a single year. We remark here that, our model and scenario reduction based methodology is applicable to handle possible extensions such as multi-stage expansion planning and demand uncertainty, and we also discuss the modifications required to handle these extensions. We first show the efficiency of the proposed solution method on the IEEE 24-bus power system and discuss the results of the $380-\mathrm{kV}$ Turkish transmission network for two cases. Different uncertainties for the generation units and AC modelling approach can also be added to the model in expense of increased number of scenarios and computational complexity in the model.

\section{References}

[1] Pereira A, Saraiva J. Generation expansion planning (GEP) - a long-term approach using system dynamics and genetic algorithms (GAs). Energy 2011;36(8):5180-99.

[2] Bakirtzis G, Biskas P, Chatziathanasiou V. Generation expansion planning by MILP considering mid-term scheduling decisions. Electric Power Syst Res 2012;86:98-112.

[3] Jadidoleslam M, Ebrahimi A. Reliability constrained generation expansion planning by a modified shuffled frog leaping algorithm. Int J Electr Power Energy Syst 2015;64:743-51.

[4] Gil E, Aravena I, Cárdenas R. Generation capacity expansion planning under hydro uncertainty using stochastic mixed integer programming and scenario reduction. IEEE Trans Power Syst 2015;30(4):1838-47.

[5] Bahiense L, Oliveira G, Pereira M, Granville S. A mixed integer disjunctive model for transmission network expansion. IEEE Trans Power Syst 2001;16(3):560-5.

[6] Teimourzadeh S, Aminifar F. MILP formulation for transmission expansion planning with short-circuit level constraints. IEEE Trans Power Syst 2016;31(4):3109-18.

[7] Li Y, McCalley J. Design of a high capacity inter-regional transmission overlay for the US. IEEE Trans Power Syst 2015;30(1):513-21.

[8] Hemmati R, Hooshmand R, Khodabakhshian A. Comprehensive review of generation and transmission expansion planning. IET Gener Trans Distrib 2013;7(9):955-64.

[9] Krishnan V, et al. Co-optimization of electricity transmission and generation resources for planning and policy analysis: review of concepts and modeling approaches. Energy Syst 2016;7(2):297-332.

[10] NERC, Definition of Adequate Level of Reliability; 2007. Accessed at 2017, June.

[11] Silva I, Rider M, Romero R, Garcia A, Murari C. Transmission network expansion planning with security constraints. IEE Proc Gener Trans Distrib 2005;152(6):828-36.

[12] Zhang H, Heydt GT, Vittal V, Quintero J. An improved network model for transmission expansion planning considering reactive power and network losses. IEEE Trans Power Syst 2013;28(3):3471-9.

[13] Rastgou A, Moshtagh J. Improved harmony search algorithm for transmission expansion planning with adequacy-security considerations in the deregulated power system. Int J Electr Power Energy Syst 2014:60:153-64.

[14] Majidi-Qadikolai M, Baldick R. Integration of N-1 contingency analysis with systematic transmission capacity expansion planning: ERCOT case study. IEEE Trans Power Syst 2016;31(3):2234-45.

[15] Majidi-Qadikolai M, Baldick R. Stochastic transmission capacity expansion planning with special scenario selection for integrating $\mathrm{N}-1$ contingency analysis. IEEE Trans Power Syst 2016;31(6):4901-12.

[16] Wu Z, Zeng P, Zhang X. Two-stage stochastic dual dynamic programming for transmission expansion planning with significant renewable generation and N-k criterion. CSEE J Power Energy Syst 2016;2(1):3-10.

[17] Choi J, et al. A method for transmission system expansion planning considering probabilistic reliability criteria. IEEE Trans Power Syst 2005;20(3):1606-15.

[18] Alizadeh B, Jadid S. Reliability constrained coordination of generation and trans mission expansion planning in power systems using mixed integer programming. IET Gener Transm Distrib 2011:5(9):948-60.

[19] Moreno R, Pudjianto D, Strbac G. Transmission network investment with probabilistic security and corrective control. IEEE Trans Power Syst 2013;28(4):3935-44.

[20] Aghaei J, Amjady N, Baharvandi A, Akbari M. Generation and transmission expansion planning: MILP - based probabilistic model. IEEE Trans Power Syst
2014;29(4):1592-601

[21] Orfanos G, Georgilakis P, Hatziargyriou N. Transmission expansion planning of systems with increasing wind power integration. IEEE Trans Power Syst 2013;28(2):1355-62

[22] Alizadeh B, Jadid S. A dynamic model for coordination of generation and transmission expansion planning in power systems. Int J Electr Power Energy Syst 2015;65:408-18.

[23] Qiu J, Dong ZY, Zhao J, Xu Y, Luo F, Yang J. A risk-based approach to multi-stage probabilistic transmission network planning. IEEE Trans Power Syst 2016;31(6):4867-76.

[24] Qiu J, Yang H, Dong ZY, Zhao J, Luo F, Lai M, Wong KP. A probabilistic transmission planning framework for reducing network vulnerability to extreme events. IEEE Trans Power Syst 2016;31(5):3829-39.

[25] Zhao JH, Foster J, Dong ZY, Wong KP. Flexible transmission network planning considering distributed generation impacts. IEEE Trans Power Syst 2011;26(3):1434-43.

[26] Özdemir Ö, Munoz FD, Ho JL, Hobbs BF. Economic analysis of transmission expansion planning with price-responsive demand and quadratic losses by successive LP. IEEE Trans Power Syst 2016;31(2):1096-107.

[27] Ugranli F, Karatepe E. Transmission expansion planning for wind turbine integrated power systems considering contingency. IEEE Trans Power Syst 2016;31(2):1476-85.

[28] Hedman K, O'Neill R, Fisher E, Oren S. Optimal transmission switching with contingency analysis. IEEE Trans Power Syst 2009;24(3):1577-86.

[29] Goldis EA, Li X, Caramanis MC, Keshavamurthy B, Patel M, Rudkevich AM, Ruiz PA. Applicability of topology control algorithms (TCA) to a real-size power system. Communication, Control, and Computing (Allerton), 2013 51st Annual Allerton Conference on. IEEE; 2013. p. 1349-52.

[30] Ruiz PA, Caramanis M, Goldis E, Keshavamurthy B, Li X, Philbrick R. Topology control algorithms (TCA) simulations in PJM with AC modeling. In: Technical Conference On Increasing Real-Time And Day-Ahead Market Efficiency Through Improved Software, Washington, FERC; 2014.

[31] Hedman KW, Oren SS, O'Neill RP. A review of transmission switching and network topology optimization. Power and Energy Society General Meeting, 2011 IEEE. IEEE; 2011. p. 1-7.

[32] Khodaei A, Shahidehpour M, Kamalinia S. Transmission switching in expansion planning. IEEE Trans Power Syst 2010;25(3):1722-33.

[33] Jabarnejad M, Wang J, Valenzuela J. A decomposition approach for solving seasonal transmission switching. IEEE Trans Power Syst 2015;30(3):1203-11.

[34] Yang Z, Zhong H, Xia Q, Kang C. Optimal transmission switching with short-circuit current limitation constraints. IEEE Trans Power Syst 2016;31(2):1278-88.

[35] Henneaux P, Kirschen D. Probabilistic security analysis of optimal transmission switching. IEEE Trans Power Syst 2016;31(1):508-17.

[36] Yu Y, Luh P. Scalable corrective security-constrained economic dispatch considering conflicting contingencies. Int J Electr Power Energy Syst 2018;98:269-78.

[37] Ardakani AJ, Bouffard F. Identification of umbrella constraints in DC-based security-constrained optimal power flow. IEEE Trans Power Syst 2013;28(4):3924-34.

[38] Ardakani AJ, Bouffard F. Acceleration of umbrella constraint discovery in generation scheduling problems. IEEE Trans Power Syst 2015;30(4):2100-9.

[39] Peker M, Kocaman AS, Kara B. Substation location and transmission network expansion problem in power system; 2017. Preprint.

[40] IEEE reliability test system. IEEE Trans Power Appar Syst 1979;98(6):2047-54.

[41] Blumsack S. Network topologies and transmission investment under electric-industry restructuring [Ph.D. thesis]. Carnegie Mellon Univ; 2006.

[42] TEIAŞ. 2013-2022 yıllar Türkiye iletim sistemi bölgesel talep tahmin ve şebeke analiz çalışmasi; 2013. Accessed at 2017, June.

[43] EPDK. Elektrik piyasası 2016 yılı piyasa gelişim raporu; 2017. Accessed at 2017, June.

[44] EPDK. 2016 yllinda uygulanacak profiller; 2016. Accessed at 2017, June.

[45] Singh A, Willi D, Chokani N, Abhari R. Optimal power flow analysis of a Switzerland's transmission system for long-term capacity planning. Renew Sustain Energy Rev 2014;34:596-607.

[46] EPDK. Proje ilerleme durumu; 2017. Accessed at 2017, June.

[47] IEA. WEO-2016 power generation assumptions; 2016. Accessed at 2017, June.

[48] Kocaman A. Essays on infrastructure design and planning for clean energy systems (Ph.D. thesis). Columbia University; 2014.

[49] TEIASS. TEIASS-Annual report 2015; 2015. Accessed at 2017, June.

[50] EPDK. Türkiye elektrik enerjisi 5 yıllık üretim kapasite projeksiyonu; 2016. Accessed at 2018, April.

[51] Wang X, Hu Z, Zhang M, Hu M. Two-stage stochastic optimization for unit commitment considering wind power based on scenario analysis. Electricity Distribution (CICED), 2016 China International Conference on. IEEE; 2016. p. 1-5.

[52] Baringo L, Conejo AJ. Wind power investment: A Benders decomposition approach. IEEE Trans Power Syst 2012;27(1):433-41. 